\title{
The Spin Studies in Few-Body Systems at Nuclotron
}

\author{
V. P. Ladygin ${ }^{1 \dagger}$, I. Dobrin ${ }^{2}$, V. V. Fimushkin ${ }^{1}$, D. A. Finogenov ${ }^{3}$, S. G. Genchev ${ }^{4}$, \\ Yu. V. Gurchin ${ }^{1}$, A. P. Ierusalimov ${ }^{1}$, A. Yu. Isupov ${ }^{1}$, K. Itoh ${ }^{5}$, M. Janek ${ }^{6}$, E. V. Karpechev ${ }^{3}$, \\ J.-T. Karachuk ${ }^{1,2}$, S. V. Khabarov ${ }^{1}$, T. Kawabata ${ }^{7}$, A. N. Khrenov ${ }^{1}$, V. A. Krasnov ${ }^{1}$, \\ A. B. Kurepin ${ }^{3}$, A. K. Kurilkin ${ }^{1}$, P. K. Kurilkin ${ }^{1}$, N. B. Ladygina ${ }^{1}$, D. Lipchinski ${ }^{2}$, \\ A. N. Livanov ${ }^{1}$, Y. Maeda ${ }^{8}$, A. I. Malakhov ${ }^{1}$, G. Martinska ${ }^{9}$, S. M. Piyadin ${ }^{1}$, J. Popovichi², \\ A. N. Prokofichev ${ }^{1}$, V. L. Rapatsky ${ }^{1}$, A. I. Reshetin ${ }^{3}$, S. G. Reznikov ${ }^{1}$, P. A. Rukoyatkin ${ }^{1}$, S. \\ Sakaguchi $^{10}$, H. Sakai ${ }^{11,12}$, Y. Sasamoto ${ }^{13}$, K. Sekiguchi ${ }^{14}$, Ya. G. Skhomenko ${ }^{15}$, K. Suda ${ }^{12}$,

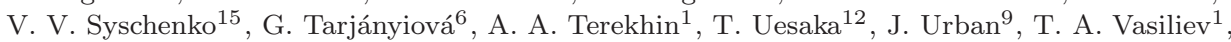 \\ I. E. Vnukov ${ }^{15}$, N. I. Zamiatin ${ }^{1}$ and E. V. Zubarev ${ }^{1}$ \\ ${ }^{1}$ Joint Institute for Nuclear Research, Dubna 141980, Russian Federation \\ ${ }^{2}$ Advanced Research Institute for Electrical Engineering, Bucharest 030138, Romania \\ ${ }^{3}$ Institute for Nuclear Research of RAS, Moscow 117312, Russian Federation \\ ${ }^{4}$ Laboratory for Technical Developments and Applications, BAS, Sofia 1784, Bulgaria \\ ${ }^{5}$ Department of Physics, Saitama University, Saitama 338-8570, Japan \\ ${ }^{6}$ Physics Department, University of Žilina, Žilina 010 26, Slovak Republic \\ ${ }^{7}$ Department of Physics, Kyoto University, Kyoto 606-8502, Japan \\ ${ }^{8}$ Department of Applied Physics, University of Miyazaki, Miyazaki 889-2192, Japan \\ ${ }^{9}$ P.J.Šafarik University, Košice 041 80, Slovak Republic \\ ${ }^{10}$ Department of Physics, Kyushu University, Fukuoka 812-8581, Japan \\ ${ }^{11}$ Department of Physics, University of Tokyo, Tokyo 113-8654, Japan \\ ${ }^{12}$ RIKEN Nishina Center, Saitama 351-0198, Japan \\ ${ }^{13}$ Center for Nuclear Study, University of Tokyo, Tokyo 113-0033, Japan \\ ${ }^{14}$ Department of Physics, Tohoku University, Sendai 980-8578, Japan \\ ${ }^{15}$ Belgorog National Research University, Belgorod 308015, Russian Federation \\ †vladygin@jinr.ru
}

Published 29 February 2016

\begin{abstract}
Recent results on spin effects in few nucleon systems obtained at Nuclotron-JINR are presented. The data on the deuteron analyzing powers in different reactions in the wide energy range demonstrate the sensitivity to the short-range spin structure of the light nuclei. The future plans on the studies with polarized deuterons from new polarized ion source at Nuclotron will be reported.
\end{abstract}

Keywords: Deuteron; elastic scattering; breakup; analyzing powers.

PACS numbers:24.70.+s, 25.10.+s, 21.45.+v

This is an Open Access article published by World Scientific Publishing Company. It is distributed under the terms of the Creative Commons Attribution 3.0 (CC-BY) License. Further distribution of this work is permitted, provided the original work is properly cited. 


\section{Introduction}

The mail goal of the Deuteron Spin Structure (DSS) experiment is to study the spin structure of two-nucleon $(2 \mathrm{~N})$ and three-nucleon $(3 \mathrm{~N})$ short-range correlations(SRC) via the measurements of the polarization observables in the deuteron induced reactions at Nuclotron. ${ }^{1-3}$

$\mathrm{SRC}$ of nucleons in nuclei is the subject of intensive theoretical and experimental works during last years. Since SRC have densities comparable to the density in the center of a nucleon which is about $\rho \sim 5 \rho_{0}\left(\rho_{0} \approx 0.17 \mathrm{fm}^{-3}\right)$, they can be considered as the drops of cold dense nuclear matter. ${ }^{4}$ These studies explore a new part of the phase diagram and very essential to understand the evolution of neutron stars.

The greate importance is the study of the spin effects in the deuteron induced reactions because they are sensitive to the SRCs spin structure. Nuclotron allows to investigate the spin effects for multi-nucleon correlations in a wide energy range. ${ }^{1-3}$

\section{Recent Results Obtained at Internal Target}

The DSS experimental program at internal target station (ITS) ${ }^{5}$ at Nuclotron ${ }^{1-3}$ is to obtain the information on the spin - dependent parts of two-nucleon $(2 N)$ and three-nucleon $(3 N)$ forces from two processes: $d p$ - elastic scattering in a wide energy range and $d p$ - nonmesonic breakup with two protons detection at energies 300-500 MeV. The motivation of this program is based on theoretical analysis of the experimental results obtained at low and intermediate energies for the deuteron induced reactions (see recent reviews ${ }^{6,7}$ and references therein).

This program at ITS was started by the measurements of the vector $A_{y}$ and tensor $A_{y y}$ and $A_{x x}$ analyzing powers in $d p$ - elastic scattering at $T_{d}$ of $880 \mathrm{MeV}^{8}$ and $2000 \mathrm{MeV} .^{9}$ The results on the angular dependencies of the analyzing powers $A_{y}, A_{y y}$ and $A_{x x}$ of $d p$ - elastic scattering obtained at Nuclotron at $880 \mathrm{MeV}^{8}$ are presented in Fig. 1. The solid, dashed and dot-dashed lines are the results of the nonrelativistic Faddeev calculations, ${ }^{6}$ relativistic multiple scattering model ${ }^{10,11}$ and optical potential approach. ${ }^{12}$ The Faddeev calculations without invoking $3 N$ forces reproduce the behaviour of the analyzing powers, however, they fail to describe the cross section data at the scattering angles larger than $70^{\circ}$ in the cms. The calculations performed within relativistic multiple scattering model ${ }^{10,11}$ describes the data on the vector analyzing power $A_{y}$ and cross section. However, there are some problems in the description of the tensor analyzing powers at large angles in the cms. The optical potential approach fails to reproduce both cross section and analyzing powers. ${ }^{8}$ The observed deficiencies in the description of the differential cross section and deuteron analyzing powers at $T_{d} \sim 880 \mathrm{MeV}$ obtained at quite large transverse momenta require the consideration of the additional mechanisms, for instance, $3 N$ forces. Since present $3 N$ forces models cannot improve the agreement with the data obtained even at lower energies, new models of $3 N$ forces (including their short-range part) should be considered. For instance, the next step in the 


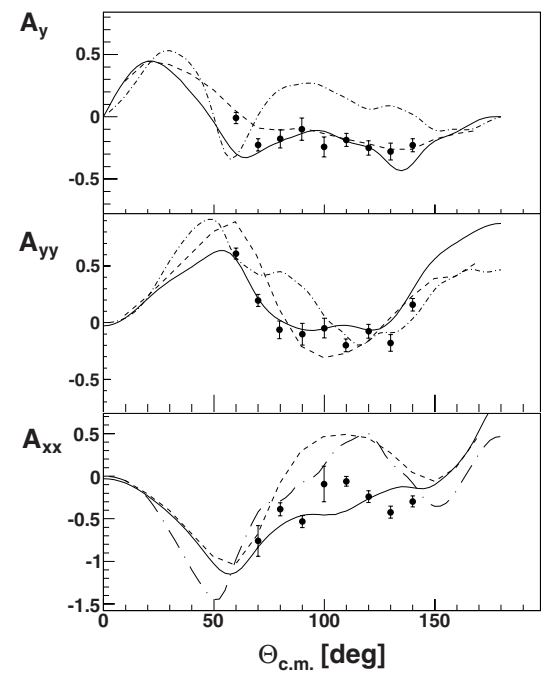

Fig. 1. The angular dependence of the analyzing powers $A_{y}, A_{y y}$ and $A_{x x}$ of $d p$ - elastic scattering at $T_{d} \sim 880 \mathrm{MeV}$. The lines are explained in the text.

relativistic multiple scattering model ${ }^{10,11}$ development could be taking into account the explicit $\Delta$ - isobar excitation.

The first stage of the DSS experimental program ${ }^{1-3}$ includes the beam energy scan of $d p$ - elastic scattering cross section at the deuteron energies $400-2000 \mathrm{MeV}$ and measurements of $d p$ - non-mesonic breakup at 300, 400 and $500 \mathrm{MeV}$ in different kinematic configurations. These measurements were performed using ITS $^{5}$ with new control and data acquisition system. ${ }^{13}$ The $10 \mu \mathrm{m} \mathrm{CH}_{2}$ foil and $8 \mu \mathrm{m}$ carbon wire were used as the targets. The effect on the hydrogen has been obtained using $\mathrm{CH}_{2}-\mathrm{C}$ subtraction. The detection apparatus for the study of $d p$ - elastic scattering consists of 4 scintillation counters with FEU-85 photomultiplier tubes for the detection of the protons and deuterons in coincidence. ${ }^{14}$ The amplitudes of the signals and timing information from the detectors were recorded and used in the further data analysis for the $d p$ - elastic scattering events selection. The scintillation counters coupled to Hamamatsu H7416MOD PMTs having better timing and amplitude resolution than FEU-85 PMTs were used for the measurements at $T_{d} \geq 1000 \mathrm{MeV}$. The $d p \rightarrow p p n$ reaction will be investigated using $\Delta E-E$ techniques for the detection of both protons. The details of the experimental setup with $8 \Delta E$ - $E$ detectors are given in ref.. ${ }^{15}$

The preliminary results on the differential cross section for the $d p$ - elastic scattering at $2000 \mathrm{MeV}$ measured at ITS with relative normalization are presented in Fig. 2 by the solid squares together with the data obtained earilier at BNL ${ }^{16}$ and Synchrophasotron ${ }^{17}$ and given by the open triangles and solid circles, respectively. One can see, a reasonable agreement of the experimental data. The dashed and 


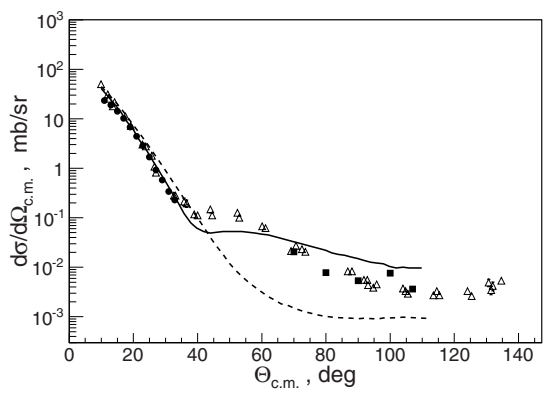

Fig. 2. The angular dependence of the $d p$ - elastic scattering cross section at $T_{d}^{l a b}=2000 \mathrm{MeV}$. Differential cross section in $d p$ - elastic scattering at $2000 \mathrm{MeV}$ obtained at ITS with relative normalization shown by the solid squares. The dashed and solid lines are the predictions of relativistic multiple scattering model taking into account single scattering only and double scattering term, respectively. The open triangles and solid circles correspond to the data obtained at $T_{d}^{l a b}=2000 \mathrm{MeV}$ in the earlier axperimenta at BNL and Synchrophasotron.

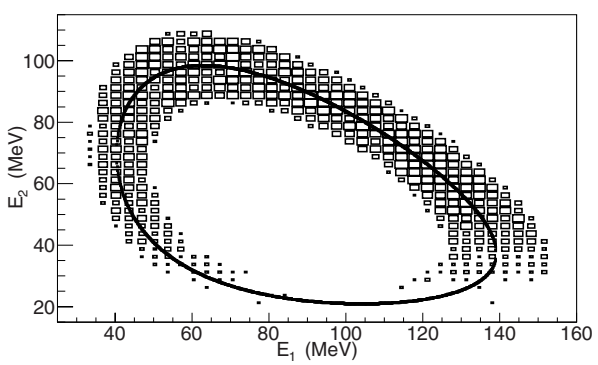

Fig. 3. The $d p \rightarrow p p n$ reaction at $T_{d}=400 \mathrm{MeV}\left(\theta_{1}=25^{\circ}, \theta_{2}=33.7^{\circ}\right.$ and $\left.\phi=44.6^{\circ}\right)$.

solid lines are the predictions of relativistic multiple scattering model ${ }^{10,11}$ taking into account single scattering only and double scattering term, respectively. The full calculations reproduce the behaviour of the experimental data on the cross section and deuteron vector analyzing power $A_{y}$ reasonably. However, this model does not describe the behaviour of the tensor analyzing powers. ${ }^{9}$

The data for different kinematic configuration for the $d p \rightarrow p p n$ reaction have been obtained at 300, 400 and $500 \mathrm{MeV}$. The preliminary results for the $d p \rightarrow p p n$ events selection obtained at $T_{d}=400 \mathrm{MeV}$ for the $\theta_{1}=25^{\circ}, \theta_{2}=33.7^{\circ}$ and $\phi=44.6^{\circ}$ are presented in Fig. 3. The data analysis is in progress.

The further progress of the DSS experimental program at ITS is related with new polarized ion source developed at LHEP-JINR. ${ }^{18}$

\section{Future Plans with Extracted Beam}

Spin physics with extracted polarized deuteron beam from new polarized deuteron source $^{18}$ can be performed with the start version of the BM@N setup. ${ }^{19}$ Since the 
multiplicity of the secondary particles is small (2 or 3 tracks) the forward and outer trackers only can be used. The BM@N experimental setup is installed at the $6 \mathrm{~V}$ beamline in the fixed-target hall of the Nuclotron. The major direction of spin studies with polarized deuteron extracted beam is the investigation of the spin-structure of light nuclei at short inter-nucleonic distances in different deuteron induced reactions, namely, $A(d, p) X, d(p, p) d, d(d, p) t\left(d\left(d,{ }^{3} \mathrm{He}\right) n\right),{ }^{3} \mathrm{He}(\mathrm{d}, \mathrm{p}){ }^{4} \mathrm{He}$ etc. in order to obtain independent information on the SRC. Another topics are the investigation of the change of the baryonic resonances properties in nuclear matter via the measurement of the polarization observables ( $T_{20}$ etc.) in the $A(d, p p) X$ and $A(d, d) X$ reactions; the investigation of the spin effects in the meson production using the $A\left(d,{ }^{3} \mathrm{He}\right) X$ reactions; the investigation of the spin effects in neutron induced reactions (with the proton spectator detection) like $n p \rightarrow p n, n p \rightarrow p p \pi^{-}$, $n p \rightarrow n p \pi^{+} \pi^{-}, n p \rightarrow d \pi^{+} \pi^{-}$etc.

The measurements of the ${ }^{3} \mathrm{He}(d, p)^{4} \mathrm{He}$ reaction is mostly challenging throughout the above mentioned experiments due to low density of the polarized ${ }^{3} \mathrm{He}$ target. ${ }^{20}$ The goal of the ${ }^{3} \mathrm{He}(d, p){ }^{4} \mathrm{He}$ reaction study at Nuclotron is to understand the reasons of the long staying puzzle, namely, the behaviour of the tensor analyzing power $T_{20}$ in $d p$ - backward elastic scattering. ${ }^{21,22}$ The experiments performed at RIKEN at the energies below $270 \mathrm{MeV}$ have shown that the polarization correlation coefficient, $C_{/ /}=1-\frac{1}{2 \sqrt{2}} T_{20}+\frac{3}{2} C_{y, y}$, for the ${ }^{3} \mathrm{He}(d, p){ }^{4}$ He reaction may be a unique probe to the D-state admixture in deuteron. ${ }^{23,24}$ The usefulness of this observable to investigate the D-state admixture is attributed to the strong spin-selectivity in neutron capture process by ${ }^{3} \mathrm{He}$ nucleus, i.e., spins of transferred neutron and ${ }^{3} \mathrm{He}$ must be anti-parallel to each other in order to form ${ }^{4} \mathrm{He}$ in the final state. In the one-nucleon exchange (ONE), the expression for $C_{/ /}$is proportional to the D-state fraction in deuteron.

The main goal of the experiment is to obtain the data on $C_{/ /}$in the energy region of $1.0-1.75 \mathrm{GeV}$, where the contribution from the deuteron D-state is expected to reach a maximum in one-nucleon exchange approximation, to obtain new information on the strange structure observed in the behaviour of $T_{20}$ in the $d p$ - backward elastic scattering and to realize experiment on the full determination of the matrix element of the ${ }^{3} \mathrm{He}(d, p)^{4} \mathrm{He}$ reaction in the model independent way. These data will help us also to understand the short-range spin structure of deuteron and effects of non-nucleonic degrees of freedom. For these purposes polarized deuteron beam from new PIS ${ }^{18}$ and spin-exchange-type polarized ${ }^{3} \mathrm{He}$ target developed at CNS of Tokyo University ${ }^{20}$ and modified for the experiment at Nuclotron can be used.

The polarization observables in the $p(d, p) d^{21,22}$ and $d(d, p) t^{25-27}$ reactions in the collinear geometry can be measured with the same experimental setup using liquid hydrogen/deuterium target or $\mathrm{CH}_{2} / \mathrm{CD}_{2}$ solid targets with carbon background subtraction. 


\section{Conclusions}

New data on the analyzing powers $A_{y}, A_{y y}$ and $A_{x x}$ in $d p$ - elastic scattering at various energies up to $2000 \mathrm{MeV}$ and well as for the $d p$ - nonmesonic breakup at the energies between 300 and $500 \mathrm{MeV}$ for different kinematic configurations can be measured at $\operatorname{ITS}^{5}$ at the Nuclotron.

First stage of the BM@N setup is well suited for the few body spin physics with polarized deuterons using new PIS. ${ }^{18}$

\section{Acknowledgments}

The work has been supported in part by the programs of the scientific cooperation between JINR and Slovak Republic in 2014 y. and by the RFBR grant No.13-0200101a.

\section{References}

1. V.P. Ladygin et al., J.Phys.Conf.Ser. 295, 012131 (2011).

2. V.P. Ladygin et al., Phys.Part.Nucl. 45, 327 (2014).

3. V.P. Ladygin et al., Few Body Syst. 55, 709 (2014).

4. L. Frankfurt, M. Sargsian, M. Strikman, Int.J.Mod.Phys. A23, 2991 (2008).

5. A.I. Malakhov et al.,Nucl.Instrum.Meth. in Phys.Res. A440, 320 (2000).

6. W. Glöckle, H. Witala, D. Hüber, H. Kamada, J. Golak, Phys.Rept. 274, 107 (1996).

7. N. Kalantar-Nayestanaki, E. Epelbaum, J.G. Messchendorp and A. Nogga, Rept.Prog.Phys. 75, 016301 (2012).

8. P.K. Kurilkin et al., Phys.Lett. B715, 61 (2012).

9. P.K. Kurilkin et al., Phys.Part.Nucl.Lett. 8, 1081 (2011).

10. N.B. Ladygina, Phys.Atom.Nucl. 71, 2039 (2008).

11. N.B. Ladygina, Eur.Phys.J. A42, 91 (2009).

12. M.A. Shikhalev, Phys.Atom.Nucl. 72, 588 (2009).

13. A.Yu. Isupov et al., Nucl.Instrum.Meth. in Phys.Res. A698, 127 (2013).

14. Yu.V. Gurchin et al., Phys.Part.Nucl.Lett. 8, 950 (2011).

15. S.M. Piyadin et al., Phys.Part.Nucl.Lett. 8, 107 (2011).

16. G.W. Bennett et al., Phys.Rev.Lett. 19, 387 (1967).

17. V.V. Glagolev, V.P. Ladygin, N.B. Ladygina and A.A. Terekhin, Eur.Phys.J. A48, $182(2012)$.

18. V.V. Fimushkin et al., Eur.Phys.J.ST 162, 275 (2008).

19. V. Ladygin et al., PoS(Baldin-ISHEPP-XXI), 038 (2012).

20. T. Uesaka et al., Nucl.Instr. and Meth. in Phys.Res. A402, 212 (1998).

21. V. Punjabi et al., Phys.Lett. B350, 178 (1995).

22. L.S. Azhgirey et al., Phys.Lett. B391, 22 (1997).

23. T. Uesaka et al., Phys.Lett. B467, 199 (1999 .

24. T. Uesaka et al., Phys. Lett. B533, 1 (2002).

25. V.P. Ladygin et al., Phys.Lett. B598, 47 (2004).

26. N.B. Ladygina, Few Body Syst. 53, 253 (2012).

27. N.B. Ladygina, Phys.Part.Nucl. 45, 187 (2014). 